\title{
On the application of the thermal conductivity equation to describe the diffusion process
}

\author{
Sofia Diakonova ${ }^{1, *}$, StepanArtyshchenko ${ }^{1}$, Daria Sysoeva ${ }^{1}$, Igor Surovtsev ${ }^{1}, \mid$ \\ and Miron Karpovich ${ }^{1}$ \\ ${ }^{1}$ Voronezh state technical University, Moskovsky prospect 14, 394026, Voronezh, Russia
}

\begin{abstract}
The paper addresses the very popular issue of diffusion of innovations. Diffusion of innovations is the last stage of the innovation process and an indicator of its success. The study of the diffusion of innovations and the application of heat conduction equations in this theory is related to the study of innovative characteristics and economic indicators that could improve the performance of enterprises, lead to profit growth and expand market coverage. The paper systematized the factors that influence the diffusion of innovations. With their help, it is possible to manage the process of diffusion of innovations in a specific market and in a certain period of time. The use of the theory and methods for solving the thermal conductivity equation allows obtaining a solution to the problem of the diffusion equation, finding the optimal parameters for creating an innovative product susceptibility on the market.
\end{abstract}

\section{Introduction}

Recently, the theory of diffusion of innovations has been of great interest in the scientific field. It finds practical application in a wide range of scientific disciplines: first of all in Economics, mass communications, sociology, social anthropology, marketing, geography, and even in medicine [1-5].

According To Schumpeter's theory of innovations, diffusion of innovations is a process of cumulative increase in the number of imitators who implement innovations after the innovator in anticipation of a more stable and high profit [6].

However, although in the economic literature, in most cases, when studying the concept of diffusion of innovations, reference is made to Y. Schumpeter, there are also earlier (and fairly well-known) studies.

The concept of diffusion of innovation is based on the theoretical developments of the French legal scholar and sociologist Gabriel tard, who in his book "The Laws of Imitation" proposed the theory of the S-curve, which describes the features of the adoption of innovations. Tard in its work also pays important attention to the factor of interpersonal communication.

The essence of this is that initially the idea is perceived by a small number of people, then their number increases, then the adoption slows down, due to the appearance of other ideas. He also introduced the concept of public opinion leadership, which means the

*Corresponding author: sof1355@yandex.ru 
influence of the most informed subjects in terms of applying innovation (who have authority on this issue) on other subjects who have less knowledge about the object.

The process of penetration (diffusion) of innovative products into markets was studied in more detail by the American scientist E. Mansfield, who confirmed the theory of G. tard. Thus, diffusion is best described by a logistic function whose graph represents the S-shaped nonlinear curve shown in Fig. 3.

Initially, positive feedback forces act, which increases the speed of diffusion of innovations. When the diffusion rate reaches a certain critical value, negative feedback is automatically activated, which causes the diffusion rate to slow down, which leads to saturation of the innovation process [7].

\section{Materials and Methods}

In the process of studying diffusion, it was found that there are certain patterns that include the innovation itself, the transfer of information from one subject to another in terms of the community or social environment, and all these components are subject to a time factor.

According to the theory of diffusion of innovations, any innovation (for example, a new idea, technique, or technology) diffuses, i.e. spreads in society according to a certain predictable model [8].

What is meant by diffusion of innovation?

Diffusion is "the process by which innovation spreads over time through certain channels among members of the social system"; innovation is "an idea, practical activity, or object whose novelty is felt by an individual or group".

The classical definition of diffusion was given by E. M. Rogers: "the Diffusion process is the distribution of a new idea from the source of invention or creation to the end user or consumption".

This definition is undoubtedly of interest, although it is given not from the standpoint of economic research of innovations, but within the framework of attributing the diffusion of innovations to the sphere of mass communications.

Diffusion of innovations is the spread over time of an innovation that has already been mastered and used in new conditions and places of application.

Diffusion leads to changes in the external environment and changes in the product itself. In the innovation chain, diffusion is not always indicated, often ignored, as is the use of marketing. Diffusion (distribution) of innovation is the basis of the modernization process. It corresponds to a phase when innovations in technologies or products have already been developed by one or more early innovators, and firms or consumers are considering adopting them [9].

Depending on the direction of diffusion, innovations can be implemented in the form of external innovations (created and implemented services, technologies, etc.) or in the form of internal innovations.

Japanese researcher Masaaki Hirooka found that the life cycle of innovations gradually decreased, starting from the time of the first industrial revolution (XVIII century) from 90 to 25 years now. The period of diffusion of innovations is expected to last about 25-30 years until the market reaches a state of maturity.

Many scientists believe that there is a lower limit and it is unlikely that in the foreseeable future it will be possible to reduce this time below 20 years! This is a mistake, there have long been studies in the field of high-tech industries about reducing the life cycles of products with a high innovative component.

It is important to note that the diffusion of innovations occurs along the logistics trajectory only under favorable economic conditions. If the economy falls into a zone of turbulence and comes to a standstill, then the diffusion is suppressed and deviates from the 
original logistics trajectory. When the economy returns to a state of recovery, the diffusion again fits into the framework of the original trajectory and makes the rest of the way, so there is no need to start from scratch [10].

Another remarkable feature of the innovation process is self-organization, which results from the nonlinear nature of innovation. Thanks to self-organization, innovations do not operate alone, but usually in groups and form so-called "clusters". Innovations within a single cluster mutually reinforce each other, causing a synergistic effect. It is due to the synergistic effect of interaction of innovations within the cluster that they cause a powerful cumulative growth of the economy, providing a breakthrough character of its development. Clusters of basic technologies lead to the emergence of new industries and, thus, launch long economic cycles, forming an upward stage of the "Kondratiev cycle", which reaches its peak at the moment of maturation of innovations. The set of clusters of basic innovations forms the technological structure [11].

The process of perception of innovation in many subjects occurs in different ways and with different time intervals. The rapid acceptance of an innovation by a large number of subjects in sociology is called a term borrowed from physics because of the great similarity "critical mass", i.e. the necessary amount of material (in physics, radioactive) needed to start a chain reaction. In our case this phenomenon appears in the economy [12]:

The critical mass is the necessary amount of information about an innovation, in which there is the greatest demand for products, and distribution can occur without the influence of marketing levers.

\section{Results}

The increase in sales directly depends on diffusion. In many cases, diffusion can be managed within the firm itself. Thus, there is a clear relationship between the diffusion of manufacturer recognition and such more" brand " concepts as commitment and loyalty [13].

In our opinion, we can also introduce the concept of "priority of the product's competitive advantages", which also affects diffusion.

Diffusion depends on a number of factors that we can use to control the diffusion of innovation [14]. These factors are shown in Fig.1. All these factors directly affect the production of a new product by the enterprise itself, as well as by imitators.

Getting commercial benefits is the main goal of commercializing innovations. It is argued that in the early stages of diffusion, there is not enough information about the relative advantages of competing innovations $[15,16]$.

However, the introduction of a continuous marketing study of all market conditions will allow you to more accurately predict the flow and speed of diffusion.

To describe the process of diffusion of innovation, you can use the well - known partial differential equation-the thermal conductivity equation (also known as the diffusion equation), its developed theory and methods for solving it, see for example.

It is necessary to override some of the variables, parameters and coefficients in accordance with the meaning of the task.

In the derivation of heat conduction equations in a natural way deals:

$U=U(M, t)$ - the temperature at the point $\mathrm{M}(\mathrm{x}, \mathrm{y}, \mathrm{z})$ a homogeneous body at a time $\mathrm{t}$.

$\mathrm{K}$ - the coefficient of internal thermal conductivity;

$\rho$ - density of matter,

$\gamma$ - the coefficient of proportionality, called the heat capacity of the substance.

When deriving the heat conduction equations, it is also convenient to consider the coefficient a, which is a function of the above coefficients $\mathrm{k}, \rho, \gamma$ :

$$
\mathrm{a}^{2}=\frac{\mathrm{K}}{\rho \gamma}
$$




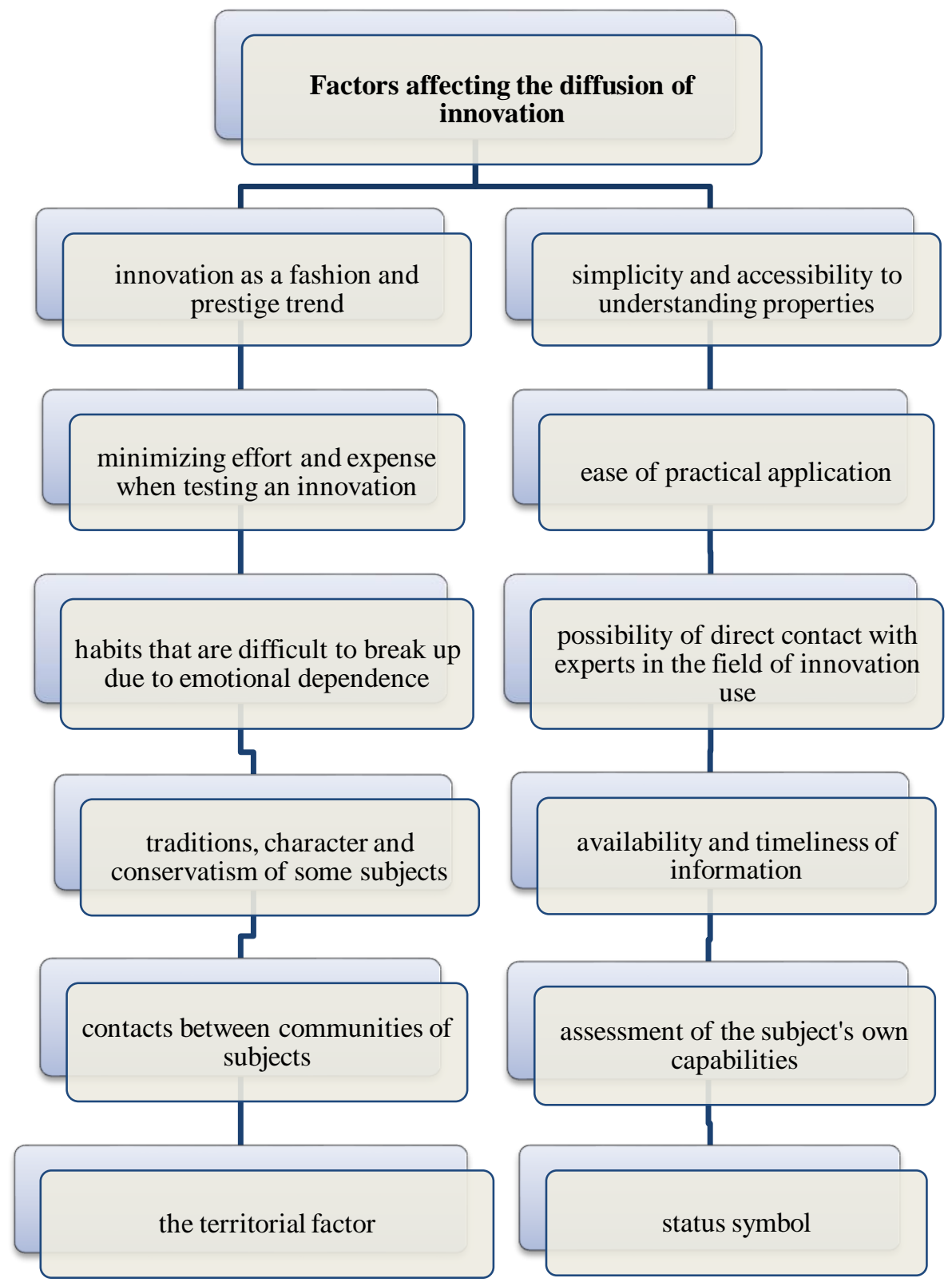

Fig.1. Factors affecting the diffusion of innovation.

In these notations, the thermal conductivity equation has the form:

$$
\frac{\partial \mathrm{u}}{\partial \mathrm{t}}=\mathrm{a}^{2}\left(\frac{\partial^{2} \mathrm{u}}{\mathrm{dx}^{2}}+\frac{\partial^{2} \mathrm{u}}{\partial \mathrm{y}^{2}}+\frac{\partial^{2} \mathrm{u}}{\partial \mathrm{z}^{2}}\right)
$$

In accordance with the meaning of the problem of finding a solution to the diffusion equation of innovations and in accordance with the closest physical analogy, we will further consider $\mathrm{k}$ as the coefficient of transfer of innovations (internal coefficient of transfer of innovations). 
$\gamma$ - we will consider it as a coefficient of proportionality, called innovation susceptibility (innovation capacity).

$\rho$ - consider it as a coefficient that determines the closeness of contacts, determines the transfer of innovations (the level of communication, the level of interpersonal communication).

In more precise accordance with the physical analogy of density $\rho$ - his is the density of connections between subjects in the process of transfer or diffusion of innovation (the density of interpersonal communication in the process of transfer or diffusion of innovation). In this case, the analog of the temperature $U=U(M, t)$ in the equation describing the diffusion of innovation is $U=U(M, t)$ - the level of innovation that changes in time and space is a quantitative indicator of the level of innovation activity, for which it is quite reasonable to introduce the concept of innovation temperature.

When solving the thermal conductivity equation, it is convenient to consider the simplified case when the body is a rod directed at the axes Ox, then the equation of thermal conductivity (and, accordingly, the equation of heat diffusion) has the form:

$$
\frac{\partial u}{\partial t}=a^{2} \frac{\partial^{2} u}{\partial x^{2}}
$$

This corresponds to the simplified case of propagation (diffusion of innovations) in time and space along only one spatial coordinate $\mathrm{x}$, i.e. the so-called one-dimensional case is considered.

For the case of an unlimited rod: the problem of finding a solution is posed $U(x, t)$ equations:

$\frac{\partial u}{\partial t}=a^{2} \frac{\partial^{2} u}{\partial x^{2}}, t>0,-\infty<x<+\infty$, satisfying the initial condition $\mathrm{U}(\mathrm{x}, \mathrm{o})=\mathrm{f}(\mathrm{x})$, $-\infty<x<+\infty$. It is solved using the Fourier method, and the solution has the form: $\mathrm{U}(\mathrm{x}, \mathrm{t})=\frac{1}{2 \mathrm{a} \sqrt{\pi \mathrm{t}}} \int_{+\infty}^{-\infty} \mathrm{f}(\xi) \mathrm{e}^{\frac{-(\xi-\mathrm{x})^{2}}{4 \mathrm{a}^{2} \mathrm{t}}} \mathrm{d} \xi$ - the Poisson integral.

Consider the following example.

Find a solution to the equation $\frac{\partial u}{\partial t}=a^{2} \frac{\partial^{2} u}{\partial x^{2}}$, for the next initial distribution of the rod temperature, in our case - the innovation temperature.

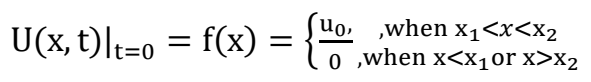

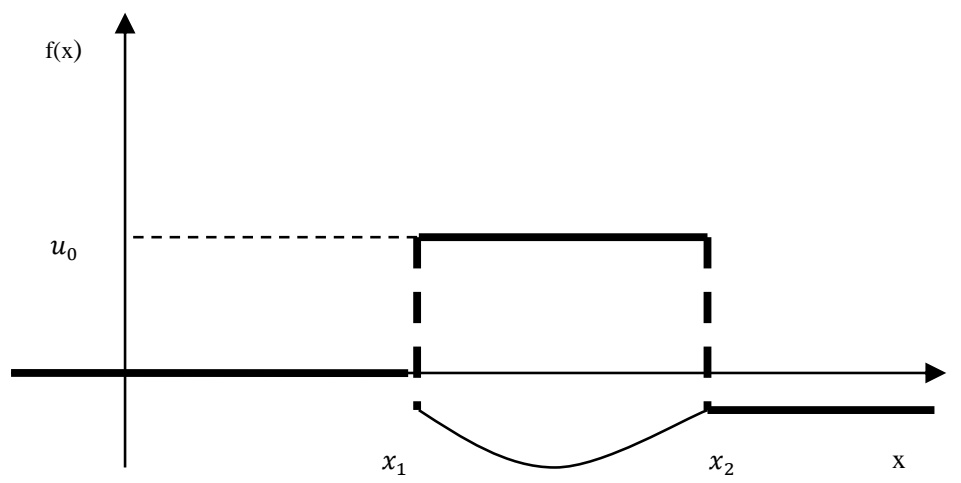

Fig.2. A graphical representation of the initial conditions satisfied by the function $U(x, t)$ in the Cauchy problem for the equation describing the diffusion of innovations.

The solution is written as a Poisson integral. 


$$
U(x, t)=\frac{1}{2 a \sqrt{\pi t}} \int_{-\infty}^{+\infty} f(\xi) e^{-\frac{(\xi-x)^{2}}{4 a^{2} t}} d \xi
$$

Since $\mathrm{f}(\mathrm{x})$ is in the interval $\left(x, x_{2}\right)$ equal to a constant temperature $u_{0}$, if the temperature is 0 outside the interval, the solution will look like this:

$$
U(x, t)=\frac{U_{0}}{2 a \sqrt{\pi t}} \int_{x_{1}}^{x_{2}} e^{-\frac{(\xi-x)^{2}}{4 a^{2} t}} d \xi
$$

This result can be converted to the probability integral (known error functionerf $\mathrm{z}$ ):

$$
\phi(z)=\frac{2}{\sqrt{\pi}} \int_{0}^{z} e^{-\mu^{2}} d \mu
$$

Assuming $\frac{\mathrm{x}-\xi}{2 \mathrm{a} \sqrt{\mathrm{t}}}=\mu ; \mathrm{d} \xi=-2 \mathrm{a} \sqrt{\mathrm{t}} \mathrm{d} \mu$ get:

$$
u(x, t)=-\frac{u_{0}}{\sqrt{\pi}} \int_{\frac{\left(x-x_{1}\right)}{2 a \sqrt{t}}}^{\frac{x-x_{2}}{2 u \sqrt{t}}} e^{-\mu^{2}} d \mu=\frac{u_{0}}{\sqrt{\pi}} \int_{0}^{\frac{\left(x-x_{1}\right)}{2 a \sqrt{t}}} e^{-\mu^{2}} d \mu-\frac{u_{0}}{\sqrt{\pi}} \int_{0}^{\frac{\left(x-x_{2}\right)}{2 a \sqrt{t}}} e^{-\mu^{2}} d \mu .
$$

Thus, the solution is expressed by the formula:

$$
u(x, t)=\frac{u_{0}}{2}\left[\phi\left(\frac{\left.x-x_{1}\right)}{2 a \sqrt{t}}-\phi\left(\frac{\left.x-x_{2}\right)}{2 a \sqrt{t}}\right)\right],\right.
$$

where $\phi(z)$-known probability integral (error function erf $z$ ).

The graph of the function $\phi(\mathrm{z})$ is the following curve:

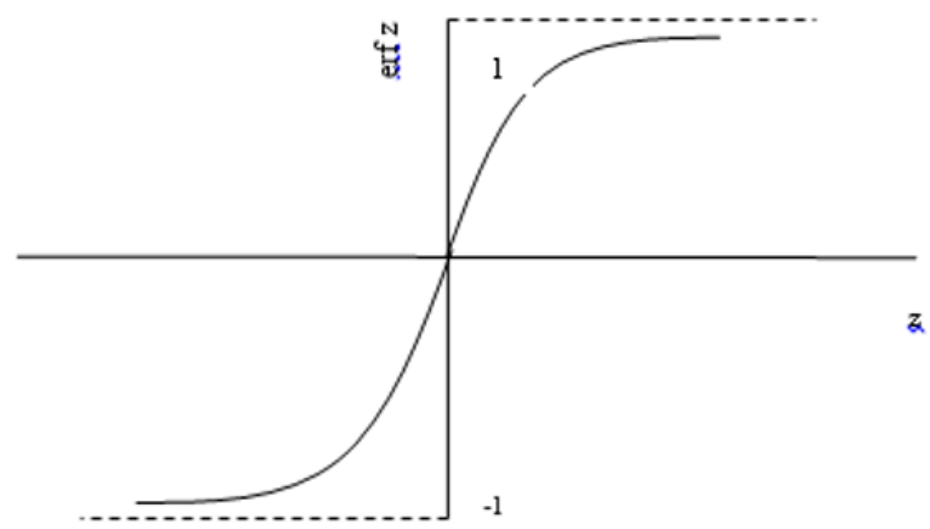

Fig. 3. Graph of the erf $\mathrm{z}$ error function.

\section{Discussions}

According to the authors, the thermal conductivity equation adequately describes the process of diffusion of innovations. This is confirmed by the fact that in the physical formulation of the problem, the actual thermal conductivity equation and the equation describing diffusion coincide. It is quite natural to introduce analogs of temperature, thermal conductivity, density, and heat capacity, such as the innovation temperature, the innovation transfer coefficient, and so on, when passing from the heat equation to the equation describing the diffusion of innovations. they are a natural and intuitive extension of their physical counterparts in the case of diffusion of innovation. The adequacy of the applied approach is also supported by the fact that the well-known logistic curve and the 
well-known error function erf $z$, which is used to Express the solution of the thermal conductivity equation, have a very similar form and properties.

\section{Conclusion}

Thus, for the analysis of propagation (diffusion of innovations), it is possible to use the classical heat conduction equation and the developed theory and methods of its solution, in particular Fourier methods. the analog of the temperature $U=U(M, t)$ in the equation describing the diffusion of innovation is $\mathrm{U}=\mathrm{U}(\mathrm{M}, \mathrm{t})$. the level of innovation changing in time and space is a quantitative indicator of the level of innovation activity, for which the authors introduced the concept of innovation temperature.

As an analogue of the $\mathrm{K}$ - coefficient of internal thermal conductivity for describing the diffusion of innovation, the authors consider the $\mathrm{K}$ - coefficient of innovation transfer.

As an analogue of the $\gamma$ - heat capacity of a substance, the coefficient $\gamma$ - the coefficient of innovative susceptibility or innovative capacity is considered.

As an analogue of the substance density, the authors introduce the density of connections between subjects in the process of transfer or diffusion of innovation.

Thus, the use of the theory and methods for solving the heat equation allows us to obtain a solution to the problem of finding a solution $U(x, t)$ - (innovation temperature) of the innovation diffusion equation (heat conduction equation) that satisfies the specified initial conditions.

The obtained solution for the simplified one-dimensional case of diffusion of innovations in time along only one spatial coordinate and for a certain particular type of initial conditions, illustrated in fig. 2, is expressed in terms of a known probability integral, namely:

$$
u(x, t)=\frac{u_{0}}{2}\left[\phi\left(\frac{\left.x-x_{1}\right)}{2 a \sqrt{t}}-\phi\left(\frac{\left.x-x_{2}\right)}{2 a \sqrt{t}}\right)\right]\right.
$$

The type of initial conditions corresponding to fig. 2, has the following meaning when considering the diffusion of innovations: the innovation temperature is 0 everywhere except for some finite interval. Under these conditions, the solution defined by formula 10 is found.

\section{References}

1. M. Raynard, The Journal of Academic Librarianship. 43, 82-86 (2017)doi: org/10.1016/j.acalib.2016.08.011

2. M.A. Douglas, R.E. Overstreet, B.T. Hazen, Business Horizons, 59, 379-389 (2016)doi: org/10.1016/j.bushor.2016.03.002

3. C. Franceschinis, M. Thiene, R. Scarpa, J. Rose, R. Cavalli, Energy, 12515, 313-326 (2017)doi: org/10.1016/j.energy.2017.02.060

4. Y. Klauzen,K.Fikhter,Environmental Innovation and Societal Transitions, 31, 64-95 (2019)doi: org/10.1016/j.eist.2019.01.003

5. S. Mannan, Sh.MdNordin, Sh. Rafik-Galea, A.R.A. Rizal, Journal of Rural Studies, 55, 316-322 (2017)doi: org/10.1016/j.jrurstud.2017.07.015

6. J. Köhler, EnvironmentalInnovationand Societal Transitions, 3, 1-15 (2012)doi: 10.1016/j.eist.2012.04.001

7. X. Zhang, International Journal of Innovation Studie,s 2, 53-64 (2018)doi: org/10.1016/j.ijis.2018.06.001 
8. S.L. Vargo, M.A. Akaka, H. Wieland, Journal of Business Research, 5 (2020)doi: org/10.1016/j.jbusres.2020.01.038

9. D. Nam, J. Lee, H. Lee, International Journal of Information Management, 49, 411-423 (2019)doi: org/10.1016/j.ijinfomgt.2019.07.017

10. R Andergassen, F Nardini, M Ricottilli, Structural Change and Economic Dynamics, 40, 72-80 (2017) doi: org/10.1016/j.strueco.2016.12.003

11. K. Kishi,European Economic Review, 118, 382-410 (2019)doi: org/10.1016/j.euroecorev.2019.05.012

12. M.E. Papazoglou, Y.E. Spanos, Research Policy, 47, 1713-1728 (2018)doi: org/10.1016/j.respol.2018.06.006

13. D.L.Florea, Procedia Economics and Finance, 23, 1527-1534 (2015)doi: org/10.1016/S2212-5671(15)00407-4

14. M.A.Halbinger, Research Policy, 47, 2028-2036 (2018) doi: org/10.1016/j.respol.2018.07.008

15. S. Kumar, B. Singh, Economic Modelling, 82, 74-86 (2019)doi: org/10.1016/j.econmod.2019.08.015

16. M. Hossain, H. Simula, M. Halme, Technology in Society, 46, 132-139 (2016) doi: org/10.1016/j.techsoc.2016.04.005 